\section{NLRP3 shapes immunity to Leishmania}

The NLRP3 inflammasome complex is assembled in response to pathogens or sterile insults and is involved in generating the proinflammatory cytokines interleukin 1 (IL-1) and IL-18. In the Journal of Clinical Investigation, Kanneganti and colleagues investigate the role of the NLRP3 inflammasome in response to infection with Leishmania major, a protozoan parasite whose control depends critically on $T$ helper type $1\left(T_{H} 1\right)$ cells. Mice deficient in a functional NLRP3 inflammasome develop a lower parasite burden after cutaneous infection. The resistance of mice with a dysfunctional NLRP3 inflammasome is associated with diminished $T_{H} 2$ responses but enhanced $T_{H} 1$ responses. IL-18 is known to augment production of the signature $T_{H} 1$ cytokine interferon- $\gamma$, but unexpectedly, in this model of $L$. major infection, IL-18 instead supports the development of $\mathrm{T}_{\mathrm{H}} 2$ cells. Accordingly, neutralization of IL-18 diminishes the L. major burden. In contrast, IL-1 seems to serve no role in modulating the $T_{H} 1$-versus $-T_{H} 2$ balance in this model. J. Clin. Invest. (17 February 2015) doi:10.1172/JCI79526

\section{Resolving Mer actions}

Prolonged inflammation can induce tissue damage; hence, regulatory mechanisms are present to dampen these responses and return to tissue homeostasis. In Science Signaling, Choi et al. show that the receptor tyrosine kinase Mer functions to downregulate the expression of inflammatory cytokines and chemokines by upregulating expression of the nuclear liver X receptors LXR $\alpha$ and LXR $\beta$ in macrophages. Blocking the interaction of Mer with its ligand Gas6 prolongs the time needed for tissue resolution of acute inflammation induced by peritoneal injection of zymosan, a TLR agonist. Gas6-Mer signaling induces kinase Aktdependent upregulation of both LXRs, which leads to the expression of pro-resolving molecules, such as TGF- $\beta$ and hepatocyte growth factor, and increases Mer expression in a positive-feedback mechanism. Therefore, the Gas6-Mer-LXR axis helps to restore tissue homeostasis after an inflammatory insult.

$L A D$ Sci. Signal. 8, ra21 (2015)

\section{Suppressive ketone}

Ketone metabolites of glycogen are produced as alternative cellular energy sources during periods of nutrient depravation or extreme exercise, processes that are associated with diminished innate immunity. In Nature Medicine, Dixit and colleagues report that the ketone $\beta$-hydroxybutyrate (BHB), which is produced by the liver during fasting, suppresses activation of the NLRP3 inflammasome in human monocytes. BHB, but not other fourcarbon ketones or short-chain fatty acids, prevents the efflux of $\mathrm{K}^{+}$ions and the formation of cytoplasmic adaptor ASC specks in response to ATP that functions as a 'signal 2' in the activation of NLRP3 after exposure to various inflammatory triggers. As a result, BHB blocks the generation and release of active IL-1 $\beta$ and IL-18. These findings help to explain how extreme caloric restriction acts to restrain innate immunity. Nat. Med. (16 February 2015) doi:10.1038/nm.3804

\section{Stressed HSCs}

The accumulation of DNA damage in hematopoietic stem cells (HSCs) is a hallmark of aging and a major contributor to functional degeneration and malignant transformation. In Nature, Walter et al. show that DNA damage in mice is a direct consequence of the exit of HSCs from their homeostatic quiescent state in response to physiological stress, such as infection or chronic blood loss. A range of stimuli that induce the entry of long-term HSCs into the cell cycle induce new DNA damage, which is dependent on enhanced mitochondrial metabolic activity and ROS production but seems to be independent of exogenous proinflammatory cytokines. Cumulative rounds of replicative stress in wild-type mice precipitate a phenotype in HSCs similar to aging, such as depletion of transplantable HSCs and a strong myeloid bias after reconstitution in recipient mice. These observations link 'stress hematopoiesis' with the functional decline of HSCs.

Nature (18 February 2015) doi:10.1038/nature14131

\section{Recruiting IRF3}

The adaptors MAVS, STING and TRIF, which transduce signals downstream of the cytosolic nucleic acid sensors RIG-I and cGAS, and the Toll-like receptors TLR3 and TLR4, specifically activate the interferon-regulatory factor IRF3. In Science, Chen and colleagues show that phosphorylation of MAVS and STING at conserved serine and threonine clusters is required for interaction with IRF3 and its subsequent phosphorylation and activation. The kinases IKK and/or TBK1, which are recruited to STING and MAVS after activation, phosphorylate these serine and threonine residues. IRF3 binds to the phosphorylated clusters through conserved, positively charged surfaces in its carboxy-terminal domain and is subsequently phosphorylated by TBK1. TRIF activates IRF3 in a similar phosphorylationdependent manner, which suggests a conserved mechanism for the selective recruitment of IRF3 and expression of type 1 interferons.

Science (29 January 2015) doi:10.1126/science.aaa2630

\section{Blocking viral maturation}

The immune response can intervene at various points in the life cycle of a virus. In Cell, Rice and colleagues screen interferon-stimulated genes to identify cell-intrinsic factors involved in resistance to influenza A virus (IAV). One such factor, the plasminogen-activator inhibitor PAI-1, substantially diminishes infection with IAV. Both IAV and interferon- $\beta$ trigger production of PAI- 1 and its release into the extracellular medium. As a final maturation step during budding, certain viruses such as IAV require cleavage of glycoproteins by host proteases. PAI-1 inhibits proteases relevant to this cleavage, such as tryptase, which results in non-infectious IAV particles. Mice deficient in PAI-1 show slightly diminished survival and worse pathology after infection with IAV. Fibroblasts derived from patients with deficiencies in the production of extracellular PAI-1 show impaired control of infection with IAV. This study therefore identifies a mechanism that controls the spread of IAV by targeting the terminal stage of the viral life cycle.

Cell (12 February 2015) doi:10.1016/j.cell.2015.01.040 